S.K. Hoon, S. Chen, F. Maloberti, J. Chen, B. Aravind: "A Low Noise, High Power Supply Rejection Low Dropout Regulator for Wireless System-on-Chip Applications"; Proc. of the IEEE Custom Integrated Circuits Conference, CICC 2005, San Josè, 21 September 2005, pp. 759-762.

(C)20xx IEEE. Personal use of this material is permitted. However, permission to reprint/republish this material for advertising or promotional purposes or for creating new collective works for resale or redistribution to servers or lists, or to reuse any copyrighted component of this work in other works must be obtained from the IEEE. 


\title{
A Low Noise, High Power Supply Rejection Low Dropout Regulator for Wireless System-on-Chip Applications
}

\author{
S. K. HOON ${ }^{(1)}$, S. CHEN ${ }^{(1)}$, F. MALOBERTI $I^{(2)}$, J. CHEN ${ }^{(3)}$, B. ARAVIND ${ }^{(1)}$ \\ ${ }^{(1)}$ Wireless Analog Technology Center, Texas Instruments, Dallas \\ ${ }^{(2)}$ Department of Electronics, University of Pavia, Italy \\ ${ }^{(3)}$ Analogic Tech, Dallas
}

\begin{abstract}
This paper presents a novel two-stage low dropout regulator (LDO) that minimizes output noise via a pre-regulator stage and achieves high power supply rejection via a simple subtractor circuit in the power driver stage. The LDO is fabricated with a standard 0.35um CMOS process and occupies $0.26 \mathrm{~mm}^{2}$ and $0.39 \mathrm{~mm}^{2}$ for single and dual output respectively. Measurement showed PSR is $60 \mathrm{~dB}$ at $10 \mathrm{kHz}$ and integrated noise is $21.2 \mathrm{uVrms}$ ranging from $1 \mathrm{kHz}$ to $100 \mathrm{kHz}$.
\end{abstract}

\section{INTRODUCTION}

Recently, there is a lot of focus on designing high performance low dropout regulator (LDO) with low noise and high PSR specification due to the wide spread popularity of hand-held products such as cellular phones and PDA (Personal Digital Assistant) [1]. The high performance LDO is commonly employed as radio frequency (RF) LDO providing quiet power supply in wireless RF system. Generally, a LDO is a closed-loop system consisting of an error amplifier, a resistive feedback network and a series pass transistor (PMOS in this case) as shown in Fig.1.

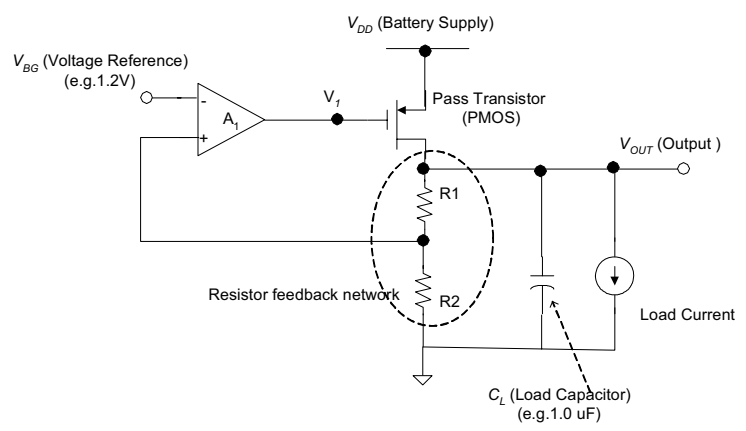

Fig.1 Conventional LDO

In Fig.1, The DC value of LDO output $\left(V_{\text {OUT }}\right)$ can be expressed as

$$
V_{\text {OUT }}=V_{B G}\left(1+\frac{R_{1}}{R_{2}}\right)
$$

where $V_{B G}$ is the voltage reference and is generally the output of a quiet voltage source such as the bandgap reference. Two specification parameters [2][3] that are usually of challenge are the power supply rejection and low integrated noise.

\section{A. Power supply rejection}

Power supply rejection (PSR) measures the LDO's ability to suppress power supply noise from its output. Assuming the contribution of supply noise due to the bandgap reference is negliglible, the small signal variations of $v_{\text {out }}$ due to supply noise $\left(v_{d d}\right)$ is given by

$$
v_{\text {out }}=A_{d d} v_{d d}+A_{1} g_{m p} r_{d s p} \beta\left(-v_{\text {out }}\right)
$$

where $A_{d d}$ is the power gain $v_{o u t} / v_{d d}$, and $A_{1}$ is the open-loop gain of the error amplifier, $\beta$ is the feedback factor $R_{2} /\left(R_{1}+R_{2}\right), g_{m p}$ and $r_{d s p}$ are the transconductance and output impedance of the pass transistor PMOS respectively.

Using the methodology as in [4], the output of LDO due to total power supply noise at low frequency can be further shown as [5]

$$
v_{\text {out }}=\left[\frac{1-A_{p 1}}{A_{1} \beta}+\frac{1}{g_{m p} r_{d s p}} \cdot \frac{1}{A_{1} \beta}\right] v_{d d}
$$

where $A_{p 1}$ is the power gain $=v_{1} / v_{d d}$. From (3), to achieve high PSR, an easy technique to improve PSR is to increase the error amplifier gain $A_{1}$ and reduce the gain factor $1 / \beta$ if possible. Alternatively, one should try to design the error amplifier such that $A_{p 1} \rightarrow 1$. For this to happen, $v_{1}$ needs to be close to $v_{d d}$ which means having the voltage at $\mathrm{V}_{1}$ tracks with the voltage at the source terminal of pass PMOS (which is connected to power supply).

\section{B. Integrated Noise}

Another important circuit performance is the total integrated noise of a LDO over the band of interest $\left(f_{2}-f_{1}\right)$. In conventional design, the total noise of the regulator is mainly contributed by different noise sources as illustrated in Fig.2. $V_{n_{-} R 1}$ and $V_{n_{-} R 2}$ are equivalent noise voltage of $R_{1}$ and $R_{2}, V_{n_{-} B G}$ is the bandgap noise and $V_{n}$ in is the input-referred noise of error amplifier itself.

The total noise power $V_{n 1}{ }^{2}$ due to the resistor feedback network is given by

$$
V_{n 1}{ }^{2}=V_{n_{-} R 1}^{2}+\left(\frac{R 1}{R 2}\right)^{2} V_{n_{-} R 1}^{2}
$$


The total noise power $V_{n 2}^{2}$ due to the noise seen at the input of LDO is given by

$$
V_{n 2}{ }^{2}=\left(\frac{1}{\beta} V_{n_{-} i n}\right)^{2}+\left(\frac{1}{\beta} V_{n_{-} B G}\right)^{2}
$$

The integrated noise $V_{n_{-} o}{ }^{2}\left(f_{2}-f_{1}\right)$ is given by

$$
\begin{gathered}
V_{n_{-} o}{ }^{2}=\int_{f 1}^{f 2}\left(V_{n 1}{ }^{2}+V_{n 2}{ }^{2}\right) d f \\
=\int_{f 1}^{f 2}\left(V_{n_{-} R 1}^{2}+\left(\frac{R 1}{R 2}\right)^{2} V_{n_{-} R 1}^{2}+\left(\frac{1}{\beta} V_{n_{-} i n}\right)^{2}+\left(\frac{1}{\beta} V_{n_{-} B G}\right)^{2}\right) d f
\end{gathered}
$$

where $1 / \beta$ is the closed-loop gain of the LDO decided by the ratio of $V_{\text {OUT }}$ and $V_{B G}\left(V_{\text {OUT }} / V_{B G}=1 / \beta\right)$. There are two ways to reduce $V_{n o}{ }^{2}$ : (a) increase the transistor's area of first stage (such as input pair) and current consumption of the error amplifier in order to reduce $V_{n 2}{ }^{2}$. (b) reduce the values of $R_{1}$ and $R_{2}$ in order to reduce $V_{n 1}{ }^{2}$. This will also result in an increase in the quiescent current consumption. In the case of system-on-chip (SoC) application, several similar LDOs can co-exist on the same chip and the increase in area and current consumption can become a serious problem.

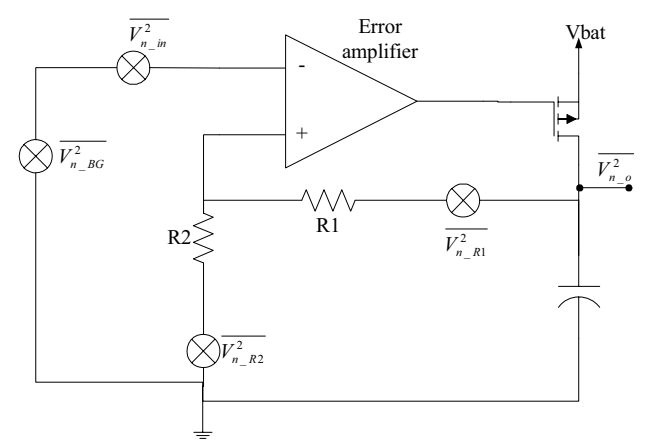

Fig.2 Noise sources of conventional LDO

\section{IMPROVED LDO}

\section{A. Power supply rejection improvement}

From (3), the PSR can be improved by having $A_{p l} \rightarrow 1$. Thus, the basic idea to improve PSR is to have an additional voltage subtractor stage as shown in Fig. 3 inserted between the pass PMOS and the error amplifier, which feeds the supply noise directly into the feedback loop and modulates the pass PMOS gate with respect to the source terminal. Note that the input terminals to the error amp need to be reversed with the addition of subtractor which would produce a phase inversion in the loop. The subtractor can be easily implemented using two NMOS transistors illustrated in Fig.3. Using a two-stage miller amplifier [4], the contribution of supply noise at $V_{2}$ would be small compared to the supply noise at diode MN1, thus $v_{2}$ (the gate voltage at MN2) $=0$. The output of subtractor stage is given by (via resistance division)

$$
v_{1}=\frac{r_{d s N 2}}{1 / g_{m N 1}+r_{d s N 2}} v_{d d}
$$

where $g_{m N 1}$ and $r_{d s N 2}$ are the transconductance of $\mathrm{N} 1$ and output impedance of $\mathrm{N} 2$ respectively. If $g_{m N 1}>g_{d s N 2}$, eq.(7) would become

$$
v_{1} / v_{d d} \approx 1 \text { or } A_{p 1} \approx 1
$$

Thus, with (3) and (8), it can be shown that the PSR of the modified LDO, is improved and given by

$$
v_{\text {out }}=\frac{1}{g_{m p} r_{d s p}} \frac{g_{m N 1}}{g_{m N 2}} \frac{1}{A_{1} \beta}
$$

Both transconductance of the NMOS transistors $\left(g_{m N 1}\right.$ and $g_{m N 2}$ ) can be made equal. This ensures the loop-gain does not increase which could jeopardize the stability of the system. Similar results can also be observed if MN1 is replaced by a PMOS MP11 with gate and drain terminals tied in a 'diode' connection. The LDO is internally compensated via $\mathrm{Cc} 1$.

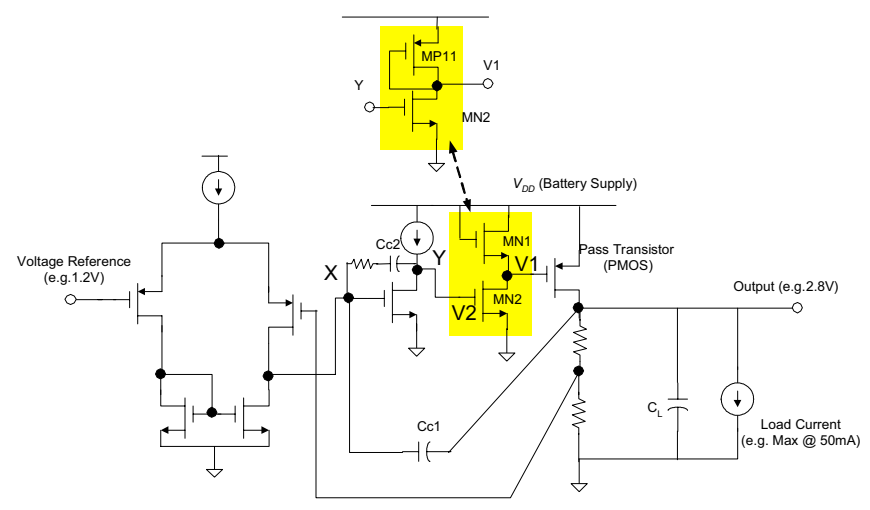

Fig.3 Implementation of PSR-boost technique

The diode connection of the subtractor provides a low impedance node $\left(1 / g_{m}\right)$ to push the parasitics pole of the pass PMOS, leaving the dominant pole due to the main miller loop (via $\mathrm{Cc} 1$ ) at node $\mathrm{X}$. Another non-dominant pole at node $\mathrm{Y}$ is pushed away by the secondary miller loop(via Cc2).

\section{B. Noise improvement}

From (6), the total noise can be improved by reducing the gain factor $1 / \beta$ and eliminating the noise term due to the resistors noise. The improvement can be made by a two-stage architecture which consists of a pre-regulator and a power driver stage as shown in Fig.4. The pre-regulator stage is formed by a reference buffer for level-shifting the bandgapreference voltage and a RC low pass filter (LPF). Note that the bandgap reference voltage is typically fixed (e.g.1.2V) and serve as general reference voltage mainly for the rest of the 
low noise modules such as baseband or RF channels. The power driver is a voltage follower configuration with a pass PMOS as its final stage so as to be able to drive the required current load. The output noise of each LDO $V_{n_{-} o}$ equals to

$$
V_{n_{-} o}{ }^{2}=\int_{f 1}^{f 2}\left(V_{n_{-} \text {in }}{ }^{2}+\left(\left|\frac{1}{s R C+1}\right| V_{n_{-} \text {pre }}\right)^{2}\right) d f
$$

where $V_{n_{-} \text {in }}$ is the input referred noise of the power driver itself and $V_{n}$ pre is the output noise of the pre-regulator. If the LPF's cut-off frequency is well below the starting frequency $f_{l}$, the second noise term in (10) can be filtered off and the final output noise can be simplified to

$$
V_{n_{-} o}{ }^{2}=\int_{f 1}^{f 2}\left(V_{n_{-} \text {in }}^{2}\right) d f
$$

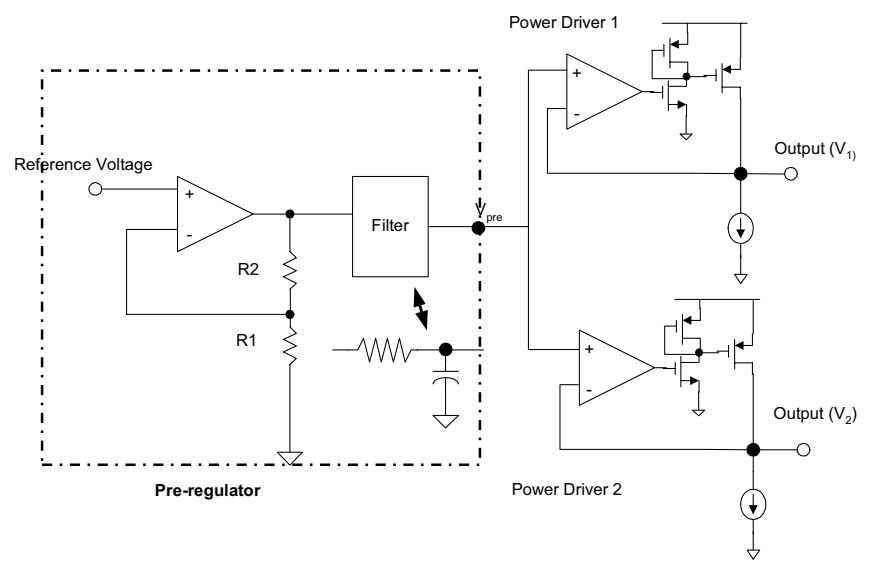

Fig.4 Proposed low noise regulators with dual output

Comparing with (6), the only noise term left in (11) is the input-referred noise of power driver. In addition, the inputreferred noise does not contain any gain factor $1 / \beta$. It is apparent that the larger the feedback factor $1 / \beta$, which means a low bandgap reference voltage and a high output voltage setting, the larger it would be the difference in noise performance.

The proposed architecture also has the following advantages: (a) LDOs with the same output voltage setting can share the same pre-regulator and LPF. The area savings are significant especially in the SoC applications where several LDOs of similar specifications are required to be on the same chip. (b). The PSR is further improved by a factor of $\beta$, since the power driver is a unity gain feedback power amplifier, the supply noise seen at output is then given by (using the subtractor stage in the power driver)

$$
v_{\text {out }}=\frac{1}{g_{m p} r_{d s p}} \frac{g_{m N 1}}{g_{m N 2}} \frac{1}{A_{1}}
$$

The filter in Fig. 4 can be implemented by a simple first-order $\mathrm{RC}$ filter. Since the density of on-chip capacitor is low (eg. $4 \mathrm{fF} / \mu \mathrm{m}^{2}$ ), normally the filter capacitor $\mathrm{C}$ is set smaller than $100 \mathrm{pF}$ to save area. Therefore, the resistance of $\mathrm{R}$ should be hundreds of Mega-ohms if the filter's cut-off frequency is set to be less than $10 \mathrm{~Hz}$. Certain CMOS processes have very high-density on-chip resistor (eg. $100 \mathrm{k} \Omega /$ square), which can further reduce the area of the filter. If such resistor is not available in the process, the circuit in [6] could be used in realizing a large resistor.

\section{EXPERIMENTAL RESULTS}

The proposed LDO is fabricated in a 0.35 um CMOS process. The chip micrograph is as shown in Fig. 5 with the left and right portion illustrate the implemenation for single and dual output respectively. Note that in the dual output case, the error amplifier is shielded with a top-plate metal to shield off any noise coupling. All measurements are performed at room temperature. The external load capacitor is $1 \mathrm{uF}$.

The noise density of the proposed LDO is plotted in Fig.6. At the condition of $V_{D D}=3.6 \mathrm{~V}$ and current load of $100 \mathrm{~mA}$, it is observed that the integrated noise of the proposed LDO is measured to be $21.2 \mathrm{uVrms}$ for frequency range from $1 \mathrm{kHz}$ to $100 \mathrm{kHz}$. The noise contribution from bandgap reference is negligible since it using an external supply with a large RC low pass filter.

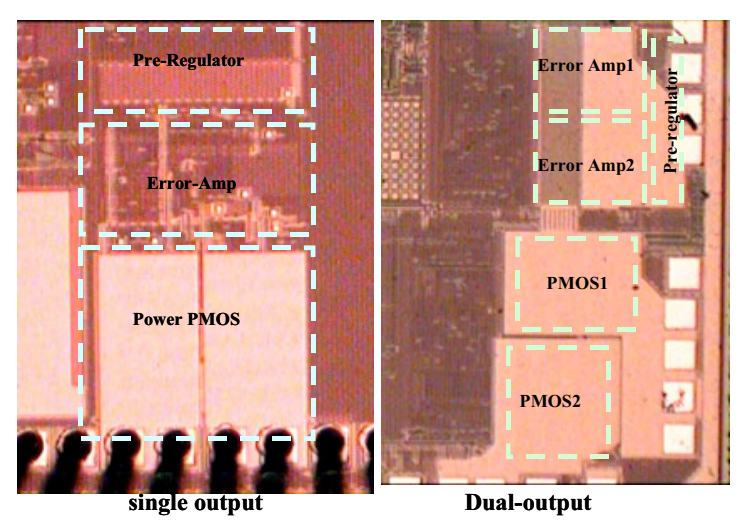

Fig.5 ChipMicrograph

For PSR measurement, $V_{D D}=3.1 \mathrm{~V}, V_{O U T}=2.8 \mathrm{~V}$, thus the dropout voltage is $300 \mathrm{mV}$. A sine-wave of $100 \mathrm{mVpp}$ is injected and the frequency is swept from $10 \mathrm{~Hz}$ to $100 \mathrm{kHz}$. The performances of this circuit have been compared with the present state-of-the-art product [7]. It is a very low-noise BJT LDO with quiescent current that is comparable with this design. The good noise performances of this design and the one described in [7] are comparable. However, this design shows a better PSR. Fig. 7 shows at least $15 \mathrm{~dB}$ improvement in a wide frequency range (up to $100 \mathrm{kHz}$ ). Another important feature is the transient response. This design is able to respond 
to current step changing from 0 to $100 \mathrm{~mA}$ in $1 \mathrm{us}$ with minimum ringing. The measured plot is shown in Fig. 8. The use of a bipolar technology gives a wide-bandwidth that made difficult for circuit compensation. Because of this, the transient response of the circuit described in [7] has a longer and ringing transient when compared with this project.

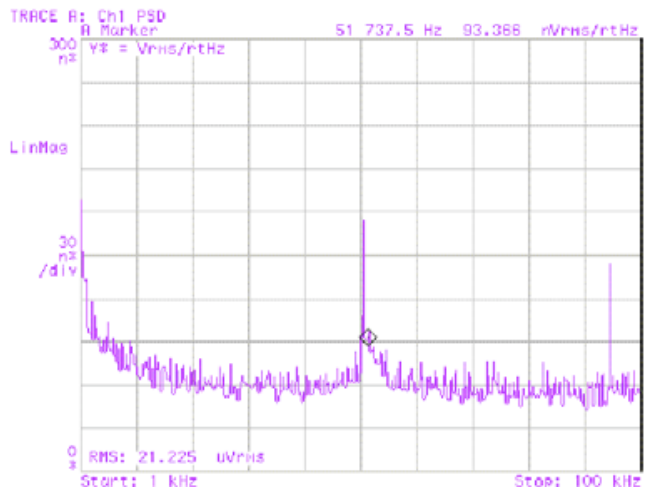

Fig.6 Noise Measurement of proposed LDO

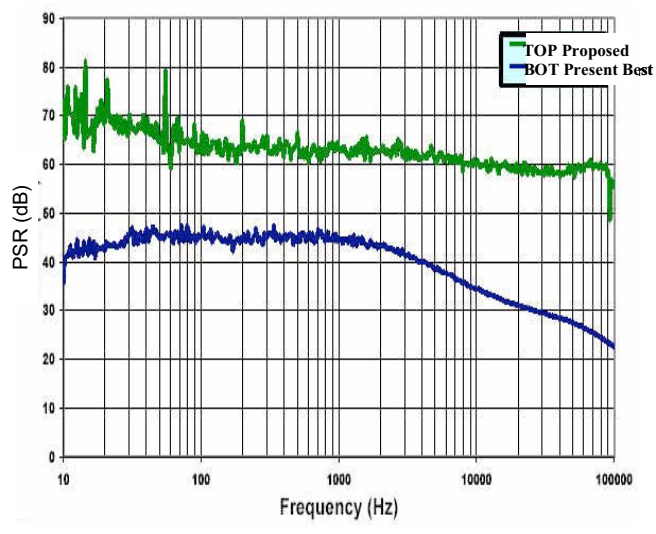

Fig.7 PSR of proposed and present best LDO

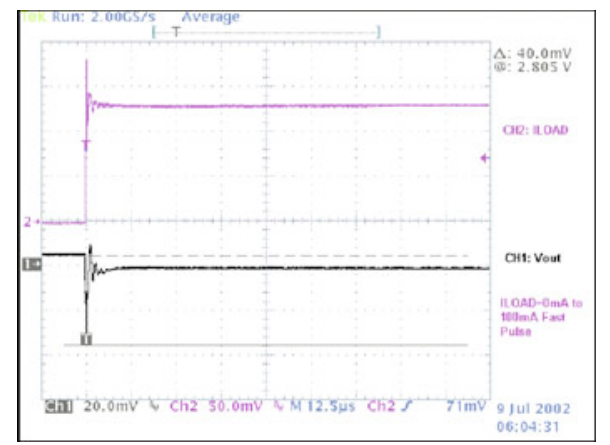

Fig 8 Transient response of proposed LDO (top : $I_{\text {OUT }}$, bottom : $V_{\text {OUT }}$ )

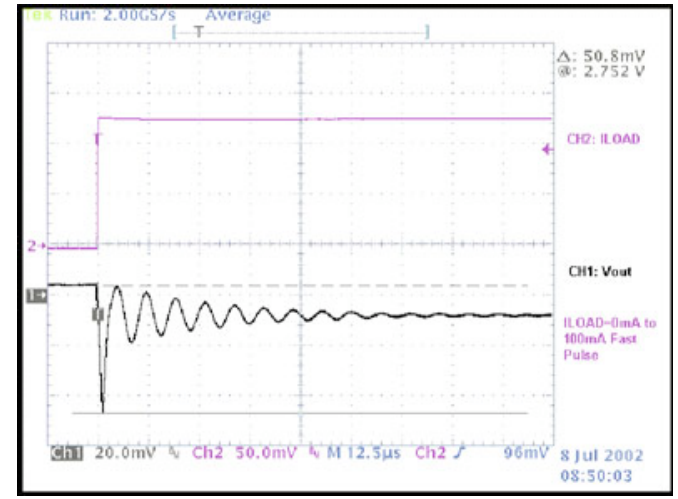

Fig.9 Transient response of present best LDO (top : $I_{\text {OUT }}$, bottom : $V_{\text {OUT }}$ )

\section{CONCLUSION}

By incorporating a pre-regulator stage and simple voltage subtractor circuit in the power driver, the LDO could achieve significant improvement in integrated noise and PSR. The proposed design does not add much complexity to the system stability compensation. It also consumes little silicon space and power, and is suitable for low voltage operation. In a wireless SoC chip, where several RF LDOs are required with similar specifications, the proposed technique could result in significant area savings by sharing the pre-regulator and have different power drivers serving different outputs which is usually for isolation purpose. The measurements are compared with the present state-of-the-art LDO. The obtained result shows that this circuit is able to obtain similar excellent noise performances but it is better in the PSR and transient control.

\section{REERENCES}

[1] D. Evans, M. McConnell, P. Kawamura and L. Krug, "SoC integration challenges for a power management/analog baseband IC for $3 \mathrm{G}$ wireless chipsets," $16^{\text {th }}$ Inl. Symp. Power Semiconductor Devices and ICs, pp. 77-80, May 2004.

[2] V. Gupta, G.A. Rincon-Mora and P. Raha, "Analysis and design of monolithic, high PSR, linear regulators for SoC applications," IEEE Intl. SoC Conf., pp. 311-315, Sept 2004.

[3] C.K. Chava and J. Silva-Martinez, "A frequency compensation scheme for LDO voltage regulators," IEEE Trans on Circuits Syst I, Vol. 51, pp. 1041-1050, Jun 2004.

[4] M.S.J. Steyart and W.MN.C. Sansen, "Power supply rejection ratio in operational transconductance amplifiers", IEEE Trans. Circuits Syst., vol.37, pp.1077-1084, 1990.

[5] S.K.Hoon, E. Yu and J. Chen, "An improved low-dropout regulator with high power supply rejection," Global Signal Processing Expo and Conf., Apr 2003.

[6] Z. Zhang, J. R. Hellums and J. M. Muza, "Low-pass filter with improved high frequency attenuation," U.S. Patent no. US6346851, Feb 2002.

[7] National Semiconductor, Micropower 150mA Low-Noise Ultra Low-Dropout Regulator, LP2985. 